\title{
Si Surface Preparation for Heteroepitaxial Growth of SiC Using in situ Oxidation
}

\author{
Li Wang ${ }^{1, a^{*}}$, Sima Dimitrijev ${ }^{1, b}$, Alan Iacopi ${ }^{1, c}$, Leonie Hold ${ }^{1, d}$, Glenn Walker $^{1, e}$, \\ Jessica Chai ${ }^{1, f}$, David Massoubre ${ }^{1, g}$ \\ ${ }^{1}$ Queensland Micro- and Nanotechnology Centre, Griffith University, Nathan, QId, 4111, Australia \\ al.wang@griffith.edu.au, bs.dimitrijev@griffith.edu.au, ca.iacopi@griffith.edu.au, \\ 'I.hold@griffith.edu.au, e glenn.walker@griffith.edu.au, fj.chai@griffith.edu.au, \\ gd.massoubre@griffith.edu.au,
}

Keywords: Silicon surface cleaning, chemical vapour deposition, silicon carbide, epitaxial growth, off-axis.

\begin{abstract}
To achieve high quality $\mathrm{SiC}$ growth on $\mathrm{Si}$ substrate, it is essential to get a smooth $\mathrm{Si}$ surface without forming $\mathrm{SiC}$ and graphitic islands during the surface cleaning and before the carbonisation process. In this paper, a novel in-situ surface cleaning method designed for the heteroepitaxial growth of $\mathrm{SiC}$ on $\mathrm{Si}$ substrate is developed using a custom-made low-pressure chemical vapour deposition reactor. The results indicate that the combination of ramping in oxygen and subsequent flowing of $\mathrm{SiH}_{4}$ avoids the contamination of $\mathrm{Si}$, enables the oxide layer to be removed smoothly, and subsequently creates a smooth Si surface with regular atomic steps. SiC grown on off-axis Si has better crystallinity and significantly smaller roughness than that grown on on-axis Si.
\end{abstract}

\section{Introduction}

Silicon surface preparation is extremely important for epitaxial film growth and other-related semiconductor processing technology. The cleaning of oxide includes ex-situ and in-situ cleaning steps. Hydrofluoric acid is widely used as an ex-situ method to remove the native oxide layer, however, a fresh Si surface easily get oxidized and contaminated by oxygen and carbon during the temperature ramping steps. In order to maintain the smoothness of the fresh Si surface and protect it from contamination, a protective oxide layer can be grown using chemical solutions before subsequent processing [1], thus, an in-situ oxide removal process is still needed to get fresh Si surface for any epitaxial processes.

The in-situ thermal desorption of the thin silicon dioxide layer starts to occur at high temperatures (as a function of oxygen pressure) [2]. However, this thermal desorption process is inhomogeneous/anisotropic: roughening and faceting of the Si surface were observed [1, 2], worse on $\mathrm{Si}(111)$ than $\mathrm{Si}(100)$. To assist the removal of oxide layer and keep a smooth Si surface, hydrogen is usually employed as atomic hydrogen can passivate the $\mathrm{Si}$ surface by forming $\mathrm{Si}-\mathrm{H}$ bonds. Since stable $\mathrm{Si}-\mathrm{H}$ bond can only exist when temperature is below $400^{\circ} \mathrm{C}$ [3], no passivation effect can be achieved beyond this temperature. However, with the aid of mobilizing Si atoms, high temperature annealing under high pressure hydrogen $\left(>10^{4} \mathrm{~Pa}\right)$ is still widely employed during the Si surface cleaning process [4], possibly because that the dynamical balance between formation and breakage of $\mathrm{Si}-\mathrm{H}$ bonds help to maintain the smoothness of the $\mathrm{Si}$ surface. High temperature annealing under high vacuum and a rapid ramp to high temperature are also used to achieve the desirable smoothness of the clean Si surface, however, when there are contamination sources present, annealing under high vacuum could not avoid the chemical reaction between the contamination source and the Si substrate. A rapid ramp to high temperature is also not practical for reactors that have a slow heating-up rate.

In this investigation, the employed custom-made low-pressure chemical vapour deposition (LPCVD) reactor has an upper pressure limit of $\sim 100 \mathrm{~Pa}$ and temperature limit of $1300^{\circ} \mathrm{C}$. The low pressure processing regime enables, a conformal $\mathrm{SiC}$ deposition on large-diameter $\mathrm{Si}$ substrates ( $\leq 300 \mathrm{~mm}$ ) by using alternating supply epitaxy (ASE) at relatively low deposition temperature [5]. It enables depositions with void-free $\mathrm{SiC} / \mathrm{Si}$ interface and precise thickness control: thickness 
adjustment at nm scale and non-uniformity less than $1 \%$. These fine controls are crucial for the fabrication of complex structures, such as SiC/AlN distributed Bragg reflector (DBR), which requires a good control of layer thickness in order to enhance the reflectance [6], making it practical for the retention of Si substrate for the low-cost GaN/Si light-emitting diode manufacture. For the growth of 3C-SiC on $\mathrm{Si}$ substrate, a carbon-containing gas has to be employed, which results in potential carbon-based contamination in the reactor. Therefore, in this paper, a unique in-situ surface cleaning process is developed for $\mathrm{Si}(111)$ wafers; the crystallinity and surface roughness of $\mathrm{SiC}$ grown at $1200^{\circ} \mathrm{C}$ on cleaned $\mathrm{Si}$ are investigated.

\section{Experiments}

The in-situ surface cleaning was performed on both on-axis (off-cut angle $<0.5^{\circ}$ ) and off-axis (off-cut angle of $3.5 \pm 0.5^{\circ}$ towards [110]) 150-mm Si(111) substrates (both are p-type doped with resistivity in the range of $1 \sim 10 \Omega \cdot \mathrm{cm}$ ) using a custom-made LPCVD reactor. Si wafers were loaded into the reactor at $600^{\circ} \mathrm{C}$ as received (without pre-treatment). The temperature was ramped up at a rate of $5^{\circ} \mathrm{C} / \mathrm{min}$ from 600 to $1000^{\circ} \mathrm{C}$ either in vacuum or under oxygen (flow rate: $100 \mathrm{sccm}$, pressure $95 \mathrm{~Pa}$ ). The native oxide formed on the Si substrate surface was removed using low pressure $\mathrm{SiH}_{4}$ at $1000 / 1200^{\circ} \mathrm{C}(<0.1 \mathrm{~Pa})$ to ensure a smooth Si surface. The surface morphology of Si was observed using scanning electron microscopy (SEM, model: JEOL JSM-6510LV) and atomic force microscopy (AFM, model: Park NX20) under non-contact mode. A carbonisation step was employed to convert the $\mathrm{Si}$ surface into $\mathrm{SiC}$ layers before the subsequent $\mathrm{SiC}$ growth (at $1200^{\circ} \mathrm{C}$ using alternating supply epitaxy), the crystallinity, surface morphology and roughness were characterised using AFM and PANalytical Empyrean x-ray diffractometry (XRD).

\section{Results and discussions}

Naturally grown oxide layer might act as an effective barrier for further reaction between carbon and Si substrate. However, once this oxide layer starts to decompose thermally during the temperature ramping (in vacuum, pressure $<0.1 \mathrm{~Pa}$ ) from 600 to $1000^{\circ} \mathrm{C}$, Si surface can roughen and chemical reactions between contamination sources and Si can occur, as shown by SEM topview images in Fig. 1 for both on-axis and off-axis Si(111) wafers, The Si surface is quite rough(the peak to valley height is around $127.78 \mathrm{~nm}$ ) with irregular saw-teeth shaped corrugated terraces, particles/islands were also seen pinned mostly around the step edges. Therefore, to get a smooth Si

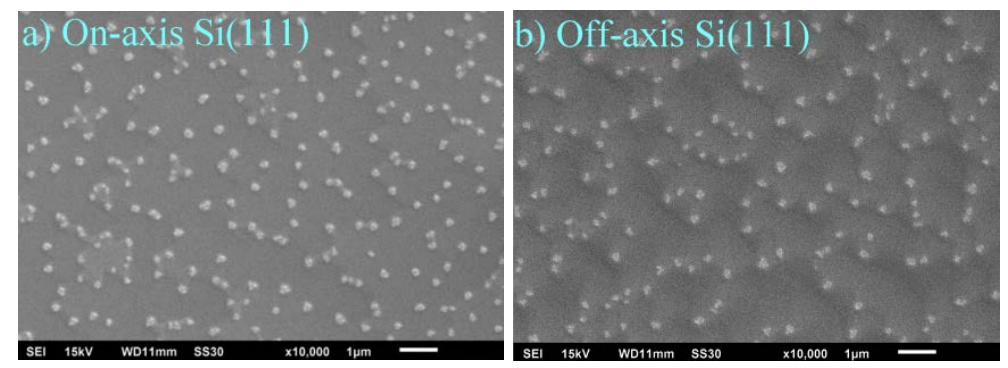

Fig. 1. SEM top-view images of on-axis and off-axis Si(111) wafers when temperature was ramped from 600 to $1000^{\circ} \mathrm{C}$ in vacuum (pressure $<0.1 \mathrm{~Pa}$ ). a) Morphology of on-axis Si, b) Morphology of off-axis Si.

surface, it is necessary to remove the oxide layer in a well-controlled manner rather than leave it thermally decomposed in vacuum. To achieve this, a high pressure of oxygen ( $95 \mathrm{~Pa}$ ) was used during the temperature ramping-up step (from 600 to $1000^{\circ} \mathrm{C}$ ) to retain the oxide layer and to react with carbon-based residues in the reactor. Upon reaching $1000^{\circ} \mathrm{C}$, an isothermal anneal under oxygen was performed for $1.5 \mathrm{~h}$ to minimise the risk of carbon-based contamination of the silicon surface. The oxide formed on the Si substrate surface was then removed using low pressure $\mathrm{SiH}_{4}$ $(1.0 \mathrm{sccm})$ at $1000^{\circ} \mathrm{C}$ or $1200^{\circ} \mathrm{C}$ to ensure a smooth $\mathrm{Si}$ surface $\left(\mathrm{SiH}_{4}\right.$ reacts with oxide to form volatile monoxide). Following the removal of oxide, the continued $\mathrm{SiH}_{4}$ flow results in a deposition of a fresh layer of Si on the activated Si substrate, the process diagram is shown in Fig. 2. The AFM 
images of the Si surface prepared in this way are shown in Fig. 3, where regular step-like features were observed on both on-axis and off-axis wafers. The root mean square (RMS) roughness for onaxis Si is around 0.17 (at $1000^{\circ} \mathrm{C}$, Fig. 3a)) and $0.13 \mathrm{~nm}$ (at $1200^{\circ} \mathrm{C}$, Fig. 3b)) over $1 \mu \mathrm{m} \times 1 \mu \mathrm{m}$ scan area, the terrace width ranges from $\sim 155$ to $\sim 322 \mathrm{~nm}$ with an average step height of $0.31 \mathrm{~nm}$ [7], indicating that $\mathrm{Si}(111)$ surface is composed of steps with uniform step height of one monolayer. The calculated off-cut angle for the on-axis Si substrate is therefore less than $0.1^{\circ}$, and no obvious step-bunching phenomenon was observed. Tiny particulates were seen evenly populating on the on-axis Si wafer surface at $1000^{\circ} \mathrm{C}$, comparatively, particle-free Si surface with regular surface steps was obtained by raising the temperature to $1200^{\circ} \mathrm{C}$. The off-axis Si has a larger RMS roughness at $1.55 \mathrm{~nm}$ over $1 \mu \mathrm{m} \times 1 \mu \mathrm{m}$ scan area (shown in Fig. 3c) and d)) for Si wafers processed at both $1000^{\circ} \mathrm{C}$ and $1200^{\circ}$. Highly parallel steps, which are normal to the wafer primary flat, have been observed. The step density on off-axis wafer is twice as high as that on the on-axis wafer, with step spacing ranged from $\sim 82 \mathrm{~nm}$ to $\sim 117 \mathrm{~nm}$ and step height varied from $6.67 \mathrm{~nm}$ to $8.33 \mathrm{~nm}$ [7], indicating that step bunching occurred during the thermal treatment.

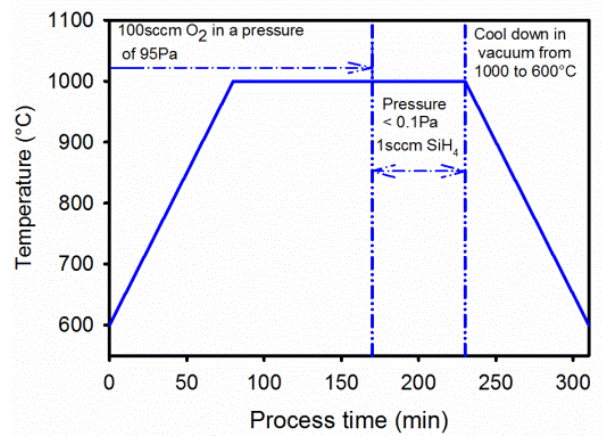

Fig. 2 Diagram of Si surface cleaning process.
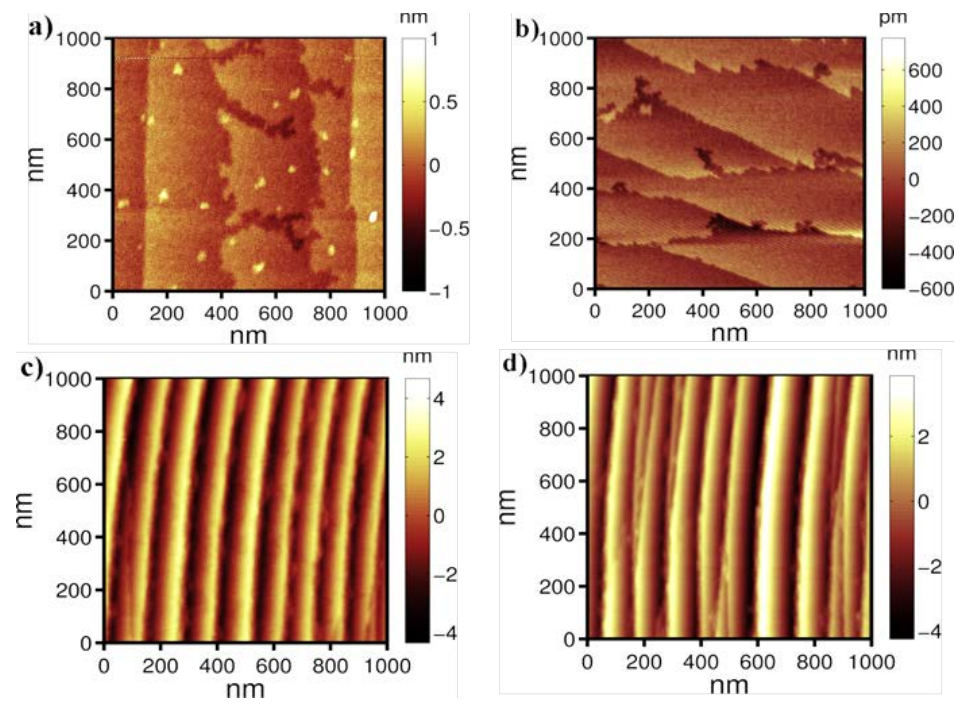

Fig. 3. Top-view AFM images of Si surface after in-situ cleaning: (a) on-axis Si prepared at $1000^{\circ} \mathrm{C}$, RMS $=0.17 \mathrm{~nm}$, (b) on-axis Si prepared at $1200^{\circ} \mathrm{C}$, RMS $=0.13 \mathrm{~nm}$ (c) off-axis Si prepared at $1000^{\circ} \mathrm{C}$, RMS $=1.55 \mathrm{~nm}$, and (d) off-axis Si prepared at $1200^{\circ} \mathrm{C}$, RMS $=1.55 \mathrm{~nm}$. The scan area is $1 \mu \mathrm{m} \times 1 \mu \mathrm{m}$.

SiC film with a thickness around $558 \pm 5 \mathrm{~nm}$ was deposited on the cleaned Si substrates, the RMS roughness from AFM measurement and FWHM of SiC(111) peak from XRD rocking curve scan are shown in Table 1. The top-view surface morphology from AFM scans is shown in Fig. 4. Triangular features were seen for $\mathrm{SiC}$ grown on on-axis $\mathrm{Si}$, while dense line features in the direction parallel to the original Si surface steps were seen for SiC grown on off-axis Si, indicating a step- 
controlled growth mode. As a result, the SiC grown on off-axis Si has better crystallinity (FWHM of $0.64 \pm 0.03^{\circ}$ ) and smaller roughness $(3.98 \pm 0.2 \mathrm{~nm})$. Both the on-axis and off-axis grown SiC have a slightly wider FWHM but smaller RMS roughness value compared to the SiC film grown by concurrent supply epitaxy [8].

Table 1 Properties of SiC films (thickness: $558 \pm 5 \mathrm{~nm}$ ) grown by ASE method at $1200^{\circ} \mathrm{C}$.

\begin{tabular}{lcc}
\hline \hline SiC sample label & $\begin{array}{c}\text { RMS roughness in a scan area } \\
\text { of } 5 \mu \mathrm{m} \times 5 \mu \mathrm{m}[\mathrm{nm}]\end{array}$ & $\begin{array}{c}\text { FWHM of rocking curve scan of } \\
\operatorname{SiC}(111) \text { peak }\left[{ }^{\circ}\right]\end{array}$ \\
\hline SiC_on-axis & $9.60 \pm 0.4$ & $0.78 \pm 0.03$ \\
\hline SiC_off-axis & $3.98 \pm 0.2$ & $0.64 \pm 0.03$ \\
\hline
\end{tabular}
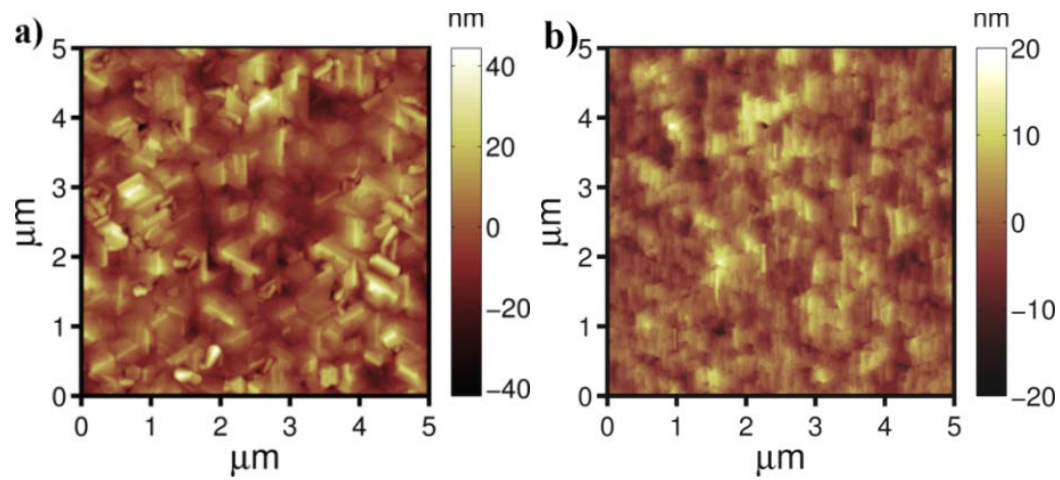

Fig. 4. Top-view AFM images of $\mathrm{SiC}(558 \pm 5 \mathrm{~nm})$ grown at $1200^{\circ} \mathrm{C}$ in a scan area of $5 \mu \mathrm{m} \times 5 \mu \mathrm{m}$ : on (a) on-axis $\mathrm{Si}$, (b) off-axis Si.

In summary, smooth Si surface with regular atomic steps was obtained on both on-axis and off-axis $\mathrm{Si}$. The combination of ramping in oxygen and subsequent flowing of $\mathrm{SiH}_{4}$ enables the oxide layer to be removed smoothly, carbon contamination is also avoided. The step density and height of a clean Si surface is dependent on the off-cut angle. The SiC film grown on off-axis Si has better crystallinity and smaller roughness due to the step-controlled growth compared to SiC grown on onaxis Si. This in-situ Si surface cleaning method is expected to be applicable to any reactors that are working under extremely low pressure range with/without potential contamination sources present in the reactor due to the previous deposition processes.

\section{Acknowledgments}

The SiC deposition, performed at Queensland Microtechnology Facility, Griffith University, Australia, was funded by SPTS Technologies and Smart Future Funds Research Partnerships Program Grant. This work was performed in part at the Queensland Node of the Australian National Fabrication Facility. The authors would like to thank Ms. Alanna Fernandes from Bluglass Ltd. Australia for performing high resolution XRD measurements.

\section{References}

[1] A. Iraji-zad, N. Taghavinia, M. Ahadian, A. Mashaei, Semicond. Sci. Technol. 15 (2000) 160.

[2] F. Smith, G. Ghidini, J. Electrochem. Soc. 129 (1982) 1300.

[3] H. Hirayama, T. Tatsumi, Appl. Phys. Lett. 54 (1989) 1561.

[4] K. Oda, Y. Kiyota, J. Electrochem. Soc. 143 (1996) 2361.

[5] L. Wang, S. Dimitrijev, J.S. Han, A. Iacopi, L. Hold, P. Tanner, H.B. Harrison, Thin Solid Films 519 (2011) 6443.

[6] D. Massoubre, R. Chu, L. Wang, J.D. Guo, J. Chai, G. Walker, L. Hold, A. Iacopi, 8th International Workshop on Nitride Semiconductors (IWN 2014).

[7] L. Wang, A. Iacopi, S. Dimitrijev, G. Walker, A. Fernandes, L. Hold, J. Chai, Thin Solid Films 564 (2014) 39.

[8] M. Katagiri, H. Fang, H. Miyake, K. Hiramatsu, H. Oku, H. Asamura, K. Kawamura, Jpn. J. Appl. Phys. 53 (2014) 05FL09. 